\title{
Mortalidade infantil no Rio de Janeiro, Brasil: áreas de risco e trajetória dos pacientes até os serviços de saúde
}

\author{
Tatiana P. Campos, ${ }^{1}$ Marilia Sá Carvalho ${ }^{2}$ \\ e Christovam C. Barcellos ${ }^{3}$
}

\begin{abstract}
RESUMO A taxa de mortalidade infantil é considerada síntese da qualidade de vida e do nível de desen volvimento de uma população. Entretanto, essa taxa é muito sensível a ações simples, como te rapia de reidratação oral, vacinação e reversão do desmame precoce, cuja cobertura tem sido ampliada. Assim, a taxa de mortalidade infantil pode não estar mais refletindo o modelo de desenvolvimento. Buscando um aprofundamento da discussão sobre a mortalidade infantil, o pre sente estudo analisou os 153 bairros do Município do Rio de Janeiro (RJ), Brasil. Nosso obje tivo foi localizar áreas de risco e grupos prioritários de intervenção que visam diminuir a mortalidade infantil no município, abordando separadamente a mortalidade neonatal e pósneonatal segundo os bairros. Além disso, foram identificados os fluxos entre o local de residên cia da criança e o local de óbito, relacionando-os à classificação socioeconômica dos bairros. A baixa freqüência de nascimentos em alguns bairros impediu a caracterização de áreas com risco de mortalidade estatisticamente significativo em relação à media do município. Cerca de um terço das mortes foram consideradas redutíveis mediante práticas adequadas de diagnóstico e tratamento. Somente $15 \%$ das causas de morte foram consideradas inevitáveis. Os componen tes da mortalidade infantil apresentaram distribuição espacial dispersa, sem uma relação direta com o perfil socioeconômico; a mudança no perfil da mortalidade infantil e a possibilidade de intervenção parece deslocar-se cada vez mais para a esfera dos serviços de saúde, especialmente os médico-assistenciais. O fluxo das crianças entre o local de residência e o local de óbito mos tra o deslocamento originado nas áreas mais pobres em direção às mais ricas, que concentram a maior parte das unidades de saúde. Essa tendência reafirma o papel fundamental do acesso à assistência médica de qualidade na determinação da mortalidade infantil.
\end{abstract}

A taxa de mortalidade infantil (TMI) é reconhecida não só como indicador

1 Secretaria Municipal de Saúde, Coordenação AP4 Rio de Janeiro, RJ, Brasil.

2 Fundação Oswaldo Cruz, Escola Nacional de Saúde Pública, Departamento de Epidemiologia e Métodos Quantitativos em Saúde. Correspondência e pedidos de separatas devem ser enviados a esta autora no seguinte endereço: Escola Nacional de Saúde Pública, Rua Leopoldo Bulhões 1480, Manguinhos, CEP 21041-210, Rio de Janeiro, RJ, Brasil. E-mail: marilia@procc.fiocruz.br

3 Fundação Oswaldo Cruz, Centro de Informação Científica e Tecnológica, Departamento de Informações em Saúde. das condições de saúde da população menor de 1 ano, mas também como a variável que melhor reflete as condições gerais de vida, sendo considerada síntese da qualidade de vida e do nível de desenvolvimento de uma população (1). Assim, é possível estabelecer relações entre a magnitude da TMI e as características do modelo de desenvolvimento (2).

Os fatores de risco para a mortalidade infantil, didaticamente, são divididos em três grupos: fatores biológi- cos, sociais e relacionados à assistência médica. Entre os fatores de risco biológicos estão: idade materna, paridade, estatura, peso antes da gravidez, ganho ponderal durante a gestação, doença materna (diabetes, hipertensão), intervalo entre os nascimentos e perdas perinatais prévias, peso ao nascer e duração da gestação. Quanto aos sociais, incluem: instrução da mãe, ocupação dos pais, renda familiar e hábitos, entre outros. Já os relacionados à assistência médica estão condiciona- 
dos a elementos como disponibilidade de recursos, aceitabilidade e resolubilidade do serviço de saúde.

Entretanto, tem-se apontado para a necessidade de rever a aplicação da TMI como indicador social completo (3). A experiência tem demonstrado que a TMI, principalmente quando situada em patamares elevados, é muito sensível a medidas simples, como a terapia de reidratação oral (TRO), a vacinação e a reversão do desmame precoce. Grandes esforços têm sido desenvolvidos no sentido de ampliar a cobertura deste tipo de ação de saúde. O próprio processo de urbanização maciça, observado em muitas partes do mundo, embora produzindo cinturões de miséria nas grandes cidades, acaba por facilitar o acesso de grandes contingentes populacionais a esses recursos (3). Dessa forma, a TMI pode não estar mais refletindo o modelo de desenvolvimento.

Um estudo realizado na Costa Rica assinala que as intervenções efetivadas na década de 70 no setor de saúde permitiram a redução da mortalidade infantil (4). Outro estudo, relacionando fatores socioeconômicos e mortalidade infantil no Equador, no período de 1970 a 1981, identificou elementos chaves, como a diminuição da natalidade e o aumento proporcional de recursos financeiros destinados ao setor de saúde, como os que mais influenciaram na redução da mortalidade infantil no país. A taxa de alfabetização foi a variável que melhor explicou as diferenças inter-regionais da TMI (5).

No Brasil, a TMI sofreu uma redução significativa, passando de 73 por mil, em 1980, para 47 por mil em 1990 e 40 por mil em 1994. Apesar da redução, a TMI continua elevada em comparação com outros países da América Latina, como Chile (13 por mil), Argentina (24 por mil) e Uruguai (19 por mil). Em relação aos países desenvolvidos (6), a diferença é ainda mais significativa: Finlândia, Japão e Suécia têm taxas de 4 por mil. No Estado do Rio de Janeiro (7) a TMI situa-se em torno de 31 por mil, com 22,4 por mil na Cidade do Rio de Janeiro em 1995.

Uma das questões fundamentais do uso da TMI como indicador de condi- ções gerais de saúde diz respeito à heterogeneidade da população, particularmente quanto a eqüidade e acessibilidade. A eqüidade pode ser entendida como a igualdade de acesso à saúde e deve ser um direito do cidadão. A acessibilidade envolve aspectos que vão mais além da assistência à saúde propriamente dita. Esta deve ser garantida do ponto de vista geográfico, através do adequado planejamento da localização dos serviços de saúde; econômico, pela remoção de barreiras derivadas do sistema de pagamento ou contribuição pelo usuário; cultural, com a adequação das normas e técnicas dos serviços ao hábitos e costumes da população em que se inserem; e funcional, mediante a oferta de serviços oportunos e adequados às necessidades da população (8).

A Cidade do Rio de Janeiro possui um padrão extremamente heterogêneo de uso do solo e de condições sociais, alternando áreas densamente povoadas com remanescentes de florestas. A localização de eventos de saúde nos bairros da cidade permite identificar associações entre estas situações geográficas e tanto o acesso a serviços de saúde como os padrões de mortalidade de diferentes grupos sociais. A incorporação do elemento geográfico à análise da distribuição dos eventos de saúde contribui para a visualização da relação entre as informações de saúde e as socioeconômicas relativas ao mesmo território, possibilitando buscar ações integradas e mais efetivas (9).

No campo da epidemiologia, os estudos ecológicos, que analisam dados agregados, têm sido revalorizados (10). Susser (11) ressalta que o contexto ambiental afeta a saúde de grupos populacionais através de processos de seleção, distribuição, interação, adaptação e outras respostas, sendo necessário medir seus efeitos no âmbito dos grupos, uma vez que as medidas em nível individual não dão conta destes processos. A avaliação do contexto ambiental é fundamental para explicar os padrões de mortalidade ou morbidade, a propagação de epidemias, a transmissão sexual de doenças, ou a transferência de comportamentos ou valores.
Os sistemas de informação do Sistema Único de Saúde (SUS), no Brasil, permitem a tabulação e o mapeamento de eventos, tendo o município como unidade espacial de análise. A desagregação dos dados para escalas locais pode desvendar desigualdades existentes nas condições de vida da população e, em geral, produz um aumento da homogeneidade interna dos indicadores. Por outro lado, pode ocorrer instabilidade estatística destes indicadores (12), uma vez que a mortalidade infantil, nesta escala, é um evento relativamente raro.

Buscando uma maior aproximação com a realidade e um aprofundamento da discussão sobre a mortalidade infantil, a escala de análise utilizada no presente estudo foram os 153 bairros do Município do Rio de Janeiro. Nosso objetivo foi localizar áreas de risco e grupos prioritários de intervenção que visam diminuir a mortalidade infantil no Município do Rio de Janeiro, abordando separadamente dois componentes segundo os bairros do município: mortalidade neonatal e pós-neonatal. Além disso, foram identificados os fluxos entre o local de residência da criança e o local de óbito, relacionandoos à classificação socioeconômica dos bairros.

\section{MATERIAIS E MÉTODOS}

Foram analisadas as TMI neonatal e pós-neonatal, bem como a mortalidade infantil proporcional por grupos de causas conforme a classificação sugerida pela Fundação Sistema Estadual de Análise de Dados (SEADE) de São Paulo (13). Esta classificação estabelece critérios de evitabilidade segundo causas básicas de morte: redutíveis por adequado controle da gravidez, redutíveis por adequada atenção ao parto, redutíveis por diagnóstico e tratamento precoce, parcialmente redutíveis e outras redutíveis.

Os dados de óbitos foram obtidos do sistema de informações sobre mortalidade (SIM), enquanto os dados de nascimento foram obtidos através do sistema de nascidos vivos (SINASC), ambos disponibilizados pela Coorde- 
nação de Programas de Epidemiologia/Subgerência de Dados Vitais da Secretaria Municipal de Saúde do Rio de Janeiro (SMS/RJ) para o ano de 1995. O mapa digital de bairros e regiões administrativas foi cedido pelo Departamento de Informações em Saúde (DIS, Fundação Oswaldo Cruz). A estes bairros foram associados dados censitários sobre habitação, estrutura etária da população e renda.

Dada a importância reconhecida das condições de vida sobre a mortalidade em menores de 1 ano e visando complementar a análise, a composição socioeconômica dos bairros foi analisada visando a construção de um perfil. Utilizou-se uma classificação multivariada (método $k$-means) sobre os seguintes indicadores: proporção (\%) de casas, definidas como construções unifamiliares; proporção de domicílios ligados à rede de esgoto; proporção de domicílios alugados; proporção de chefes com renda mensal acima de 10 salários mínimos; e proporção de setores censitários favelados em relação ao total de setores de cada bairro. Os bairros foram classificados em cinco grupos de acordo com o perfil socioeconômico, identificados de A a E, cujo perfil está apresentado na tabela 1 .

Os histogramas dos componentes da TMI permitiram identificar saltos na distribuição de freqüência, através dos quais foram estabelecidos empiricamente três pontos de corte. As taxas de mortalidade neonatal e pós-neonatal foram divididas em quatro categorias: baixa, média, pouco alta e alta. Estas categorias estão representadas nos gráficos junto com a linha mediana (figura 1). Os pontos de corte para a mortalidade neonatal foram: baixa, de 0 a 3,99 por 1000 nascidos vivos; média, de 4 a 19,99; pouco alta, de 20 a 31,99; e alta, igual ou acima de 32 por 1000 nascidos vivos. Para a mortalidade pós-neonatal, os pontos de corte foram: mortalidade baixa, de 0 a 3,99 por 1000 nascidos vivos; média, de 4 a 12,99; pouco alta, de 13 a 19,99; e alta, igual ou acima de 20.

A ocorrência do óbito em menores de 1 ano ( $n=2777)$, em 1995, analisada para os 153 bairros, foi modelada através da distribuição de Poisson, com

TABELA 1. Proporção média de indicadores socioeconômicos (\%) e número de bairros por agrupamento, Rio de Janeiro (RJ), Brasil, 1995

\begin{tabular}{|c|c|c|c|c|c|c|}
\hline \multirow[b]{2}{*}{ Agrupamento } & \multirow[b]{2}{*}{ Casas $^{a}$} & \multicolumn{4}{|c|}{ Domicílios Renda > 10} & \multirow{2}{*}{$\begin{array}{c}\text { Número de } \\
\text { bairros }\end{array}$} \\
\hline & & Esgoto $^{b}$ & Alugados & Salários $^{\mathrm{C}}$ & Favela & \\
\hline$A$ & 18 & 96 & 28 & 39 & 6 & 28 \\
\hline$B$ & 57 & 92 & 32 & 10 & 11 & 58 \\
\hline C & 80 & 83 & 22 & 5 & 39 & 36 \\
\hline$D$ & 77 & 16 & 18 & 15 & 63 & 7 \\
\hline$E$ & 89 & 6 & 15 & 7 & 13 & 24 \\
\hline$R^{2} /\left(1-R^{2}\right)$ & 3 & 13 & 0,5 & 2 & 1 & \\
\hline
\end{tabular}

FIGURA 1. Distribuição dos componentes da mortalidade infantil por bairros, Rio de Janeiro (RJ), Brasil, 1995
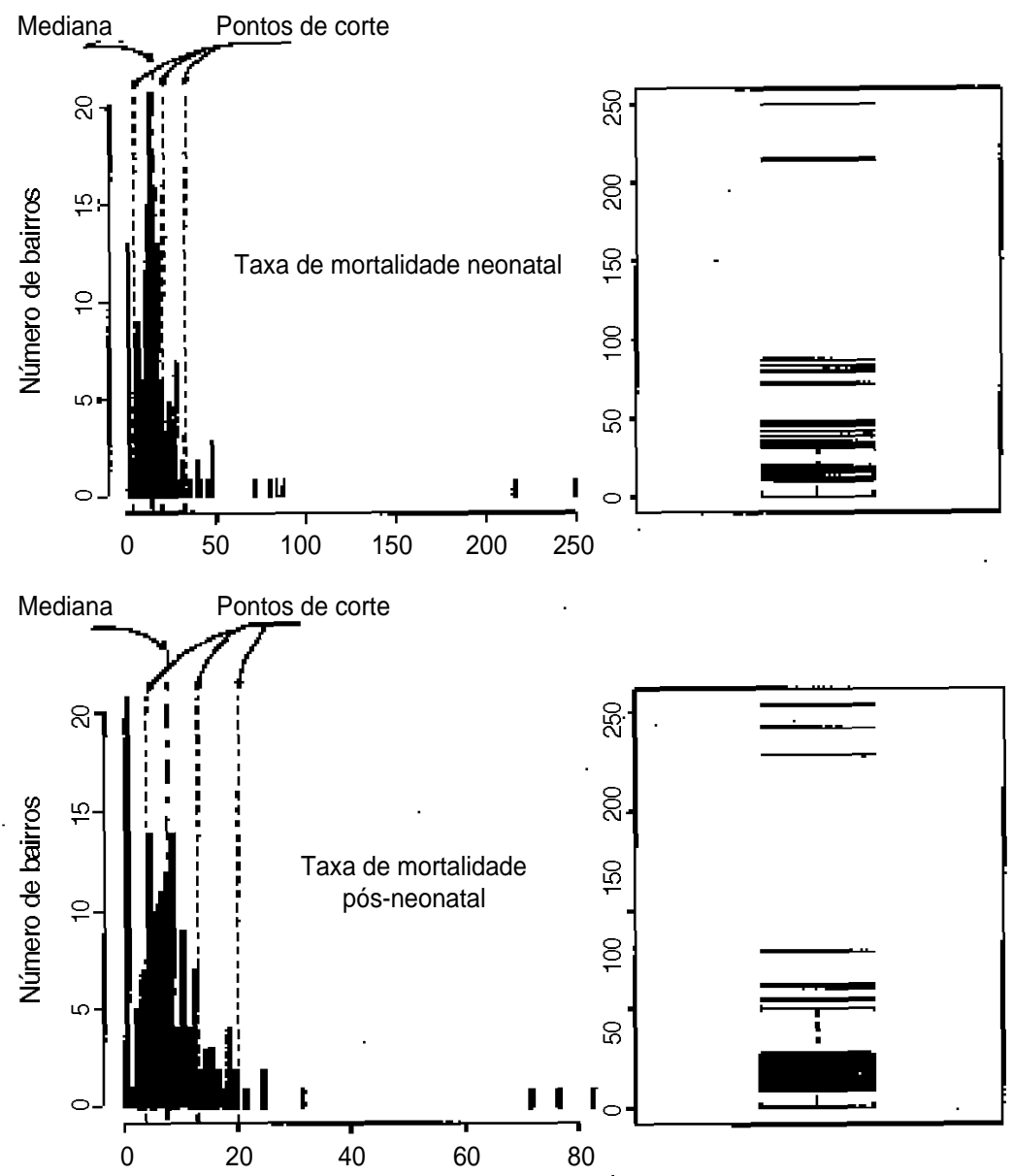

significância de 0,05, gerando oito categorias para os valores: baixo significativo, baixo não-significativo, médio significativo, médio não-significativo, pouco alto significativo, pouco alto não-significativo, alto significativo, alto não-significativo. Tal análise permitiu destacar os bairros que apresentavam TMI significativamente diferente da TMI média do município. 
Na identificação do fluxo de crianças entre o local de residência da mãe e o local do óbito, a unidade de análise foi formada pelas 26 regiões administrativas (RA). As RA são formadas por um conjunto de bairros e são territórios de gestão do sistema de saúde local. Foram excluídos os fluxos menores do que seis crianças para a análise da mortalidade neonatal e os fluxos menores do que três para a mortalidade pós-neonatal. A largura de cada flecha é proporcional ao fluxo de óbitos (onde $\mathrm{n}=69$ foi o maior fluxo na mortalidade neonatal e $\mathrm{n}=35$ na pósneonatal). $\mathrm{O}$ volume dos fluxos internos, ou seja, aqueles óbitos ocorridos dentro da própria RA de residência, é apresentado utilizando um símbolo (triângulo) na mesma escala das setas.

\section{RESULTADOS}

O sistema de informações sobre mortalidade (SIM) processou 2777 declarações de óbitos (DO) de menores de 1 ano em 1995. Dessas crianças, $76 \%$ residiam no Município do Rio de Janeiro. A TMI para o município como um todo foi de 21,8 por mil, sendo a TMI neonatal de 14,4 por mil e a pós-neonatal, de 7,3 por mil.

A análise dos campos do SIM permitiu a avaliação da disponibilidade e qualidade dos dados. Com exceção das variáveis idade, sexo, tipo de gestação, residência e local de ocorrência do óbito, foi alta a proporção de informações ignoradas para as demais variáveis. A informação sobre variáveis importantes para a análise socioeconômica, como a ocupação da mãe e a escolaridade da mãe e do pai, era ignorada em 24,30 e $45 \%$, dos registros respectivamente. O não preenchimento do peso ao nascer foi de $29 \%$. Não houve informação sobre a idade da mãe e o número de filhos nascidos mortos em 28 e $9 \%$ dos registros, respectivamente.

A tabela 2 apresenta a distribuição de causas básicas de óbitos segundo a classificação da SEADE. Como se vê, cerca de $80 \%$ dos óbitos são evitáveis. Tal informação contribui para a compreensão do padrão de distribuição espacial da mortalidade infantil por bairros, parti-
TABELA 2. Distribuição de óbitos infantis segundo classificação da Fundação Sistema Estadual de Análise de Dados (SEADE), Rio de Janeiro (RJ), Brasil, 1995

\begin{tabular}{llr}
\hline \multicolumn{1}{c}{ Classificação dos óbitos } & \multicolumn{1}{c}{ Códigos da CID 9 } & $\%$ \\
\hline Redutíveis por adequado controle da gravidez & $090,760,773,037,771.3$ & 1 \\
Redutíveis por adequada atenção ao parto & $762,763,766,767,768,771$ & 7 \\
& exceto 771.3 e 771.8 & \\
Redutíveis por diagnóstico e tratamento precoces & $771.8,520-579,770,772,774$, & 29 \\
& $777,680-709,775,776,778$ & \\
Parcialmente redutíveis por adequado controle & $260-269,764,765,769$ & 17 \\
$\quad$ da gravidez & & \\
Outras redutíveis & $001-009,276,460-519$ & 11 \\
Não evitáveis & $140-239,390-459,740-759$ & 15 \\
Desconhecida & $779,780-796,798,799$ & 9 \\
Outras & Restante da CID & 10 \\
\hline
\end{tabular}

FIGURA 2. Mapa da taxa de mortalidade neonatal e nível de significância por bairros, Rio de Janeiro (RJ), Brasil, 1995

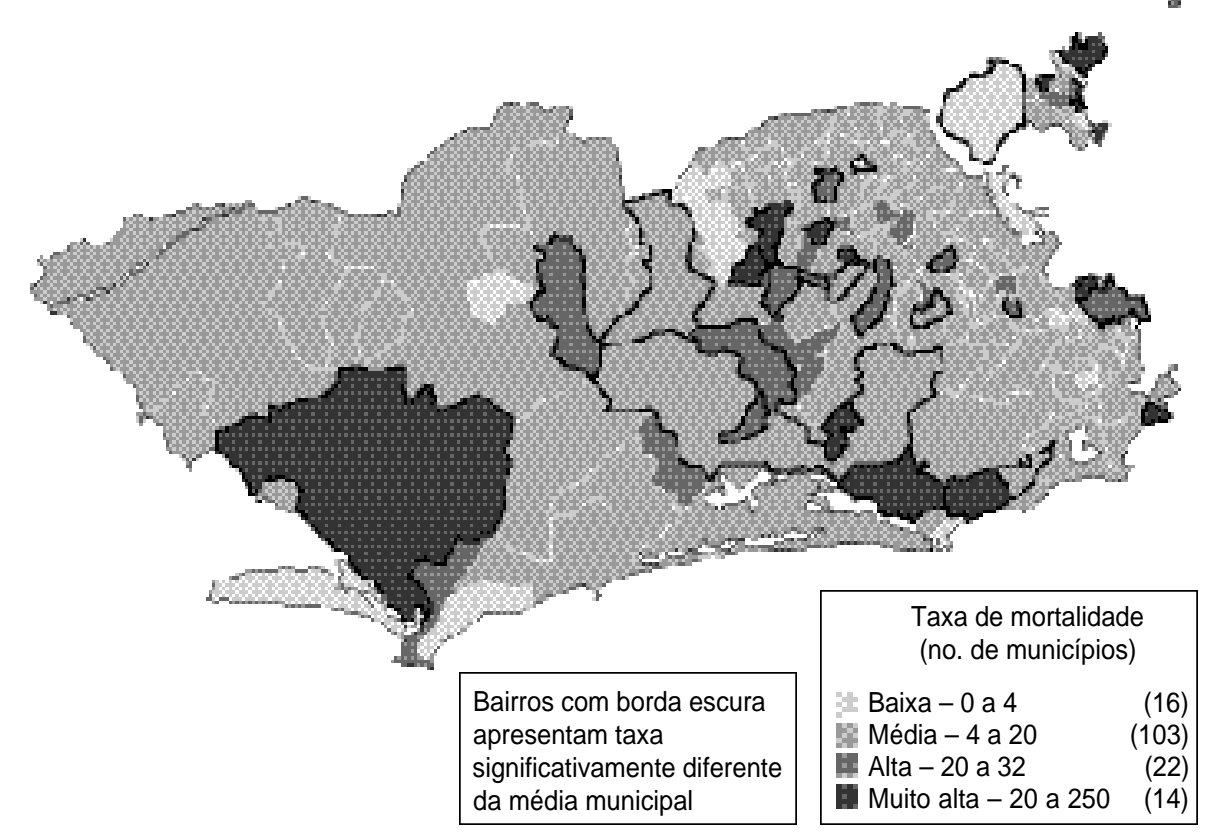

cularmente quando se analisa o fluxo das crianças entre o local de residência e o local do óbito. O agrupamento proposto pela SEADE permite relacionar a evitabilidade dos óbitos infantis e o acesso aos serviços de saúde.

A distribuição de freqüência da taxa de mortalidade neonatal por bairros (figura 1) mostra uma grande variabilidade de valores, com muitos valores zero. A caixa do gráfico mostra uma distribuição concentrada em valores baixos, com a mediana em torno de 10 por mil, e um grande número de valo- res marginais no extremo superior da distribuição. A caixa do gráfico da taxa de mortalidade pós-neonatal apresenta distribuição menos concentrada, com a mediana ainda mais baixa e um menor número de valores excessivamente altos.

A figura 2 mostra o mapeamento da taxa de mortalidade neonatal por bairros e a figura 3 , a taxa de mortalidade pós-neonatal. Para a TMI pós-neonatal, a diferença em relação à média do município foi significativa para os sete bairros que apresentaram taxas mais 
altas. Pode ser observada uma coincidência de posições dos bairros quanto aos dois componentes da mortalidade infantil. Os bairros com valores extremos (altos ou baixos) de TMI neonatal também se enquadravam no mesmo grupo para a mortalidade infantil pósneonatal. $\mathrm{O}$ mapeamento de causas específicas de óbitos por bairros, não apresentado aqui, não permitiu identificar nenhum padrão de distribuição espacial, pois, dado o pequeno número de óbitos em cada grupo, os valores esperados situaram-se próximos a zero.

A figura 4 permite identificar os fluxos de óbitos neonatais entre a RA de residência da mãe e de ocorrência do óbito. Nota-se uma tendência de deslocamento de crianças com menos de 1 mês no sentido oeste-leste, em direção ao centro da cidade, confirmando que as RAs onde há disponibilidade de serviços de saúde com perfil de atendimento para gestantes de risco - concentradas na zona sul e centro - possuem um poder de atração em relação aos óbitos. Esse padrão é semelhante ao fluxo de gestantes entre o local de residência e o local do parto, analisado por Campos e Carvalho (14). Tal relação se torna evidente quando se superpõem os fluxos à classificação socioeconômica dos bairros: as áreas que se caracterizam pela importação pertencem aos grupos A e B, que dispõem de maior infra-estrutura urbana. $\mathrm{O}$ mapa da figura 4 permite também observar que, na maioria das RA, os fluxos intra-RA são muito pequenos se comparados aos inter-RA, evidenciando, em grande parte das RA, a insuficiência de recursos para o atendimento da demanda local.

A figura 5 apresenta os fluxos de crianças com idade entre 1 mês e 1 ano. O fluxo para esta faixa etária manteve a direção predominante oeste-leste e apresenta as seguintes diferenças em relação à mortalidade neonatal: também foram observados fluxos em direção ao extremo oeste do município; os fluxos originados da zona oeste tiveram um padrão mais disperso em relação ao destino, embora algumas áreas na zona sul e centro tenham permanecido com forte poder de atração.
FIGURA 3. Mapa da taxa de mortalidade pós-neonatal e nível de significância por bairros, Rio de Janeiro (RJ), Brasil, 1995

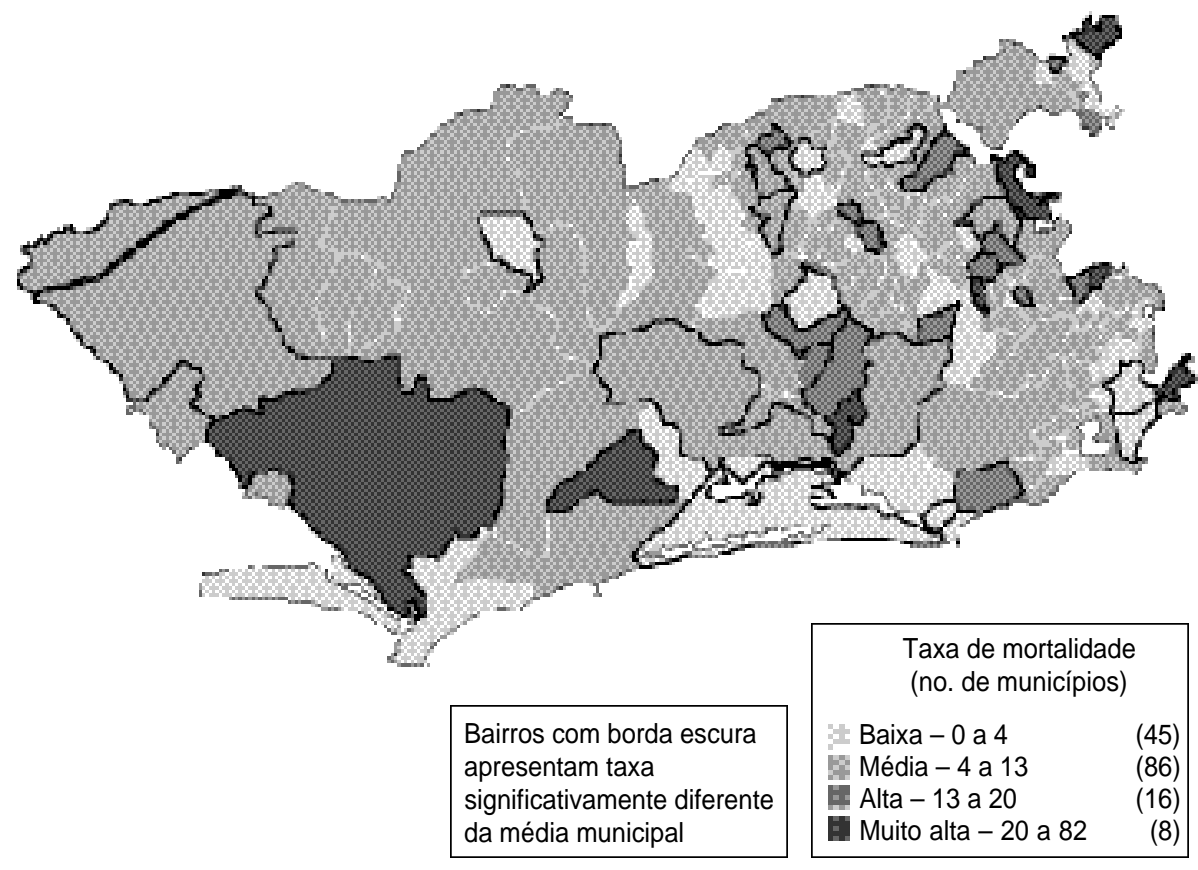

FIGURA 4. Fluxo de crianças menores de 1 mês entre a região administrativa de residência e a região administrativa de óbito sobreposto ao padrão da classificação socioeconômica multivariada por bairros, Rio de Janeiro (RJ), Brasil, 1995

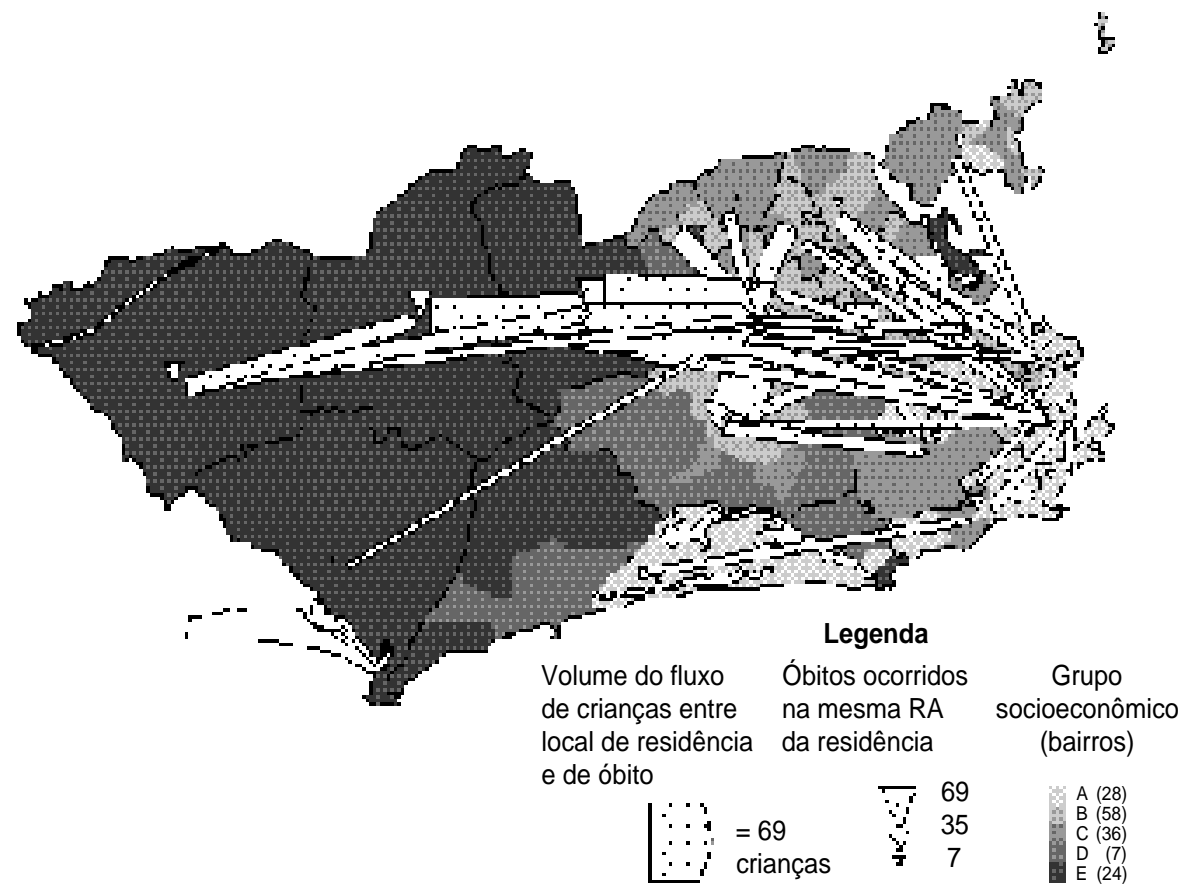


FIGURA 5. Fluxo de crianças de 1 mês a 1 ano entre a região administrativa de residência e a região administrativa de óbito sobreposto ao padrão da classificação socioeconômica multivariada por bairros, Rio de Janeiro (RJ), Brasil, 1995

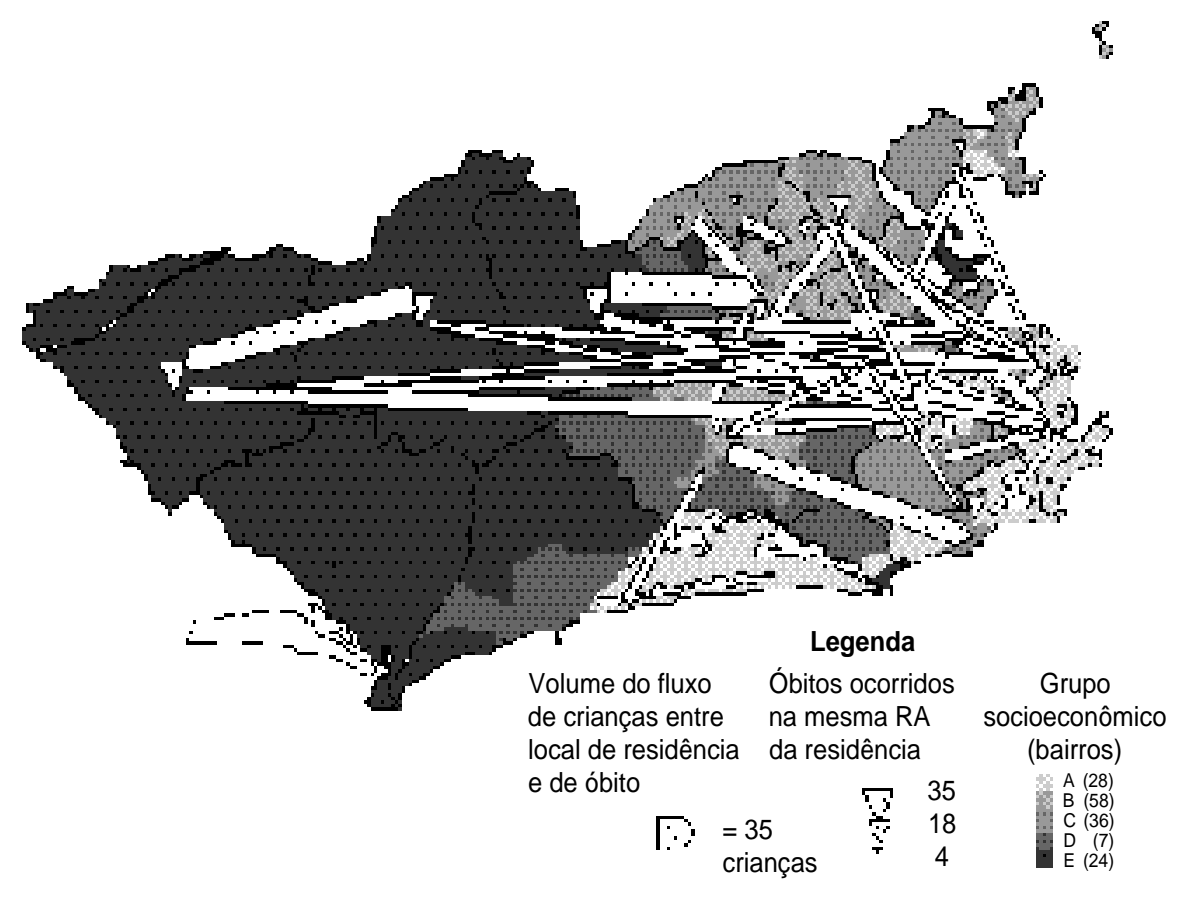

\section{DISCUSSÃO}

A alta proporção de informações ignoradas prejudicou uma análise mais detalhada da mortalidade infantil segundo características dos pais e da criança. Mesmo no caso dos óbitos neonatais precoces, que ocorrem, em sua maioria, no mesmo estabelecimento onde ocorreu o nascimento, o percentual de informação ignorada foi elevado. É importante citar que uma via da declaração de nascidos vivos (DN) fica anexada ao prontuário e que todas as variáveis que devem ser preenchidas na DO de menores de 1 ano constam na DN, de forma que esta poderia ser consultada no caso de óbito neonatal.

A análise da taxa de mortalidade foi realizada a partir da comparação entre o valor calculado em cada bairro e o seu valor esperado, com base na média do município. A significância desta diferença depende tanto da diferença entre neiro que, através de análise espacial da TMI segundo os municípios, identificou a perda da associação da mortalidade infantil com variáveis sociais, ao comparar as taxas de mortalidade infantil e indicadores socioeconômicos derivados do censo demográfico de 1980 com os mesmos indicadores para o censo de 1991. A conclusão do estudo é que a mudança no perfil da mortalidade infantil e a possibilidade de intervenção desloca-se cada vez mais para a esfera dos serviços de saúde, especialmente os médico-assistenciais (15). Também D'Orsi (16), utilizando a mesma unidade de análise - bairros - para analisar o perfil de nascidos vivos, não identificou relação espacial para a variável "proporção de recémnascidos com baixo peso", considerada como principal fator preditivo de risco para a mortalidade infantil. A variável "proporção de recém-natos com índice de Apgar acima de 8", muito mais relacionada às condições de assistência ao parto do que às condições de vida em geral, foi a que apresentou forte estrutura espacial (16). Evidentemente, também o acesso à assistência é fruto de uma realidade socioeconômica. Porém, é necessário que se busquem novos indicadores capazes de medir esta variável.

A capacidade de um indicador de detectar populações de risco varia conforme o local e a escala de análise. Em situações onde o diferencial socioeconômico reflete-se diretamente no aporte nutricional da gestante, a incidência de baixo peso ao nascer será maior, gerando incremento também na mortalidade infantil. Por outro lado, ainda que tenham sido identificados bairros onde as condições de vida são mais precárias, o impacto destas na mortalidade infantil vem diminuindo ao longo das últimas décadas, graças, inclusive, aos programas de mitigação como reidratação oral, vacinação e atendimento às infecções respiratórias agudas. Em cidades como o Rio de Janeiro, onde as condições de saúde da gestante não são significativamente desiguais entre os bairros, a detecção dos grupos de maior risco pode estar relacionada a outros indicadores. 

assim, pelas suas características epidemiológicas, menos um indicador de risco agregado e mais um evento "sentinela" de uma ocorrência potencialmente evitável. Outros estudos permitiriam aprofundar esta análise, seja diminuindo a escala e investigando a ocorrência de baixo peso em macrorregiões com grandes contrastes (por exemplo, municípios da Região Sudeste), seja aumentando a escala e investigando microáreas (setores censitários). No caso do Rio de Janeiro, esta abordagem traria, possivelmente, uma contribuição importante, determinada pelas características da cidade, onde os bairros são compostos por populações heterogêneas, com a presença de favelas "intercaladas" com áreas urbanizadas. Tal estudo poderia detectar grupos particularmente vulneráveis, nos quais ainda é grande o risco de morrer antes de completar 1 ano, como é o caso de famílias de rua ou de áreas extremamente carentes na expansão das favelas.
A mortalidade infantil torna-se,

O mapa de fluxo de pacientes, ao contrário da taxa de mortalidade, mantém forte relação com o índice socioeconômico. Bairros com condições socioeconômicas desfavoráveis tendem a "exportar" crianças para atendimento em outras áreas mais favorecidas da cidade. Este fluxo mostra apenas a origem (local de residência) e destino (unidade de saúde onde ocorreu o óbito infantil), não demonstrando a trajetória das mães em busca de atendimento para seus filhos. Se antes, com o filho no ventre, este "périplo" era longo, agora, com o filho no colo, é ainda mais complexo. Observa-se também que os deslocamentos identificados no mapa de fluxo para a mortalidade pós-neonatal são maiores se comparados à mortalidade neonatal. A maior dispersão dos destinos, no caso da mortalidade pósneonatal, demonstra que se o sistema de referência e contra-referência para o parto é precário e limitado, é ainda mais precário para o menor de 1 ano gravemente doente. Este tipo de abordagem demonstra a necessidade da

\section{REFERÊNCIAS}

1. Paim JS, Dias CN, Araújo JD. Influência de fatores sociais e ambientais na mortalidade infantil. Bol Oficina Sanit Panam 1980; 88(4): 327-40.

2. Belizzi ALM. Características da mortalidade infantil na coorte de nascimentos de 1992 no Município de Niterói (Rio de Janeiro) [dissertação de mestrado]. Rio de Janeiro: Instituto de Medicina Social da Universidade Estadual do Rio de Janeiro; 1996.

3. Beccker RA. Análise de mortalidade: delineamentos básicos. Brasília: Ministério da Saúde/Fundação Nacional de Saúde, Coordenação de Informações Epidemiológicas; 1991.

4. Rosero Bixby L. Determinantes socioeconómicos y sanitarios del descenso de la mortalidad infantil. Bol Ofic Sanit Panam 1985;99(5): 510-527.

5. González Pérez G, Gálvez González AM, Jaramillo Villarreal M. Factores socioeconómicos y mortalidad infantil en Ecuador, 19701981. Rev Saude Publica 1988;22(4): 273-280.

6. Fundo das Nações Unidas para a Infância. Situação mundial da infância. Brasília: UNICEF; 1996.
7. Theme MM. Mortalidade infantil no município do Rio de Janeiro - 1995. Saúde em Foco 1996;5(14):25-27.

8. Unglert CVS. O Enfoque da Acessibilidade no Planejamento da Localização e Dimensão de Serviços de Saúde. Rev Saude Publica 1990; 24(6):445-452. ment of environmental health indicators. In: Briggs D, Corvalán $C$, Nurminen $M$, eds. Linkage methods for environment and health analysis. Genebra: World Health Organization; 1996. p. 19-53.

10. Castellanos PL. On the concept of health and disease. Description and explanation of the health situation. Epidemiol Bull 1990;10(4): $1-8$.

11. Susser M. The logic in ecological: I - The logic of analysis. Am J Public Health 1994; 84(5):825-829.

12. Carvalho MS, Cruz OG. Análise espacial por micro-áreas: métodos e experiências. In: Veras $\mathrm{RP}$, ed. Epidemiologia contextos e pluralidade. Rio de Janeiro: Editora FIOCRUZ/
9. Corvalán C, Briggs D, Kjellstrom T. Develop- discussão de oferta e acesso de grupos específicos da população a serviços de emergências pediátricas, isto é, a normatização de um sistema regionalizado e hierarquizado de serviços de saúde. Enquanto em países desenvolvidos discute-se a condução do parto e o monitoramento de crianças cada vez mais prematuras, no Brasil ainda vemos crianças em boas condições de nascimento irem a óbito por falta de condições mínimas de assistência médica.

Algumas sugestões se impõem a título de conclusão deste trabalho. Em primeiro lugar, a estruturação de um adequado sistema de referência e contra-referência para a assistência ao parto e à criança. Além disso, a utilização dos bancos de dados secundários como instrumentos de avaliação da qualidade da assistência orientaria, de imediato, a implantação de um sistema de supervisão e avaliação regulares, particularmente das maternidades conveniadas com o SUS, o que contribuiria para reverter, rapidamente, o quadro aqui apresentado.
ABRASCO: 1998. p. 79-89. [Série Epidemiológica no 4.]

13. Fundação SEADE. A mortalidade infantil em São Paulo no primeiro semestre de 1990. Conjuntura Demográfica 1991;14/15:35-151.

14. Campos TP, Carvalho MS. Perfil de nascimento e óbitos infantis: a busca da assistência no Município do Rio de Janeiro. Cad Saude Publica 1999;16(2):109-118.

15. Szwarcwald CL, Leal MC. Característica da mortalidade neonatal no Estado do Rio de Janeiro na década de 80: uma visão espaçotemporal. Rev Saude Publica 1997;31(5): 457-465.

16. D'Orsi E. Perfil de nascimentos no município do Rio de Janeiro: uma análise espacial. Cad Saude Publica 1998;14(2):367-379.

Manuscrito recebido em 15 de setembro de 1999. Aceito em versão revisada em 16 de maio de 2000 . 
ABSTRACT The infant mortality rate has been considered a summary of the quality of life and level of development of a given population. However, this indicator is very sensitive to such simple measures as oral rehydration therapy, vaccination, and continuation of breast-feeding. Given that such health activities have become more widespread, an infant mortality rate may no longer reflect a particular development model. With the aim of broadening the discussion regarding infant mortality, this study analyzed the 153 neighborhoods of the city of Rio de Janeiro, Brazil. Our objective was to identify areas with more risk, and the priority groups for interventions to decrease infant mortality. We analyzed neonatal and postneonatal mortality in each neighborhood. We also identified the children's home neighborhood and the location of their deaths and related these results to the socioeconomic classification of the corresponding neighborhoods. In relation to the average infant mortality rate for the city, we could not make statistically significant comparisons for some neighborhoods due to their small number of births. One-third of the infant deaths could have been prevented with early diagnosis and treatment. Only 15\% of the deaths were considered unavoidable. Both neonatal mortality and postneonatal mortality were geographically dispersed, with no direct association with the socioeconomic profile of the neighborhoods. An analysis of the children's place of residence and the location of their deaths showed flows of patients from poor areas to more affluent city areas with better health services. This pattern highlights the effect of access to quality medical care on infant mortality. 